\title{
Role of Rab GTPases in Hepatocellular Carcinoma
}

\author{
Chun-Cheng Yang ${ }^{1, *}$ \\ Guang-Xiao Meng '** \\ Zhao-Ru Dong $\mathbb{D}^{1, *}$ \\ Tao $\mathrm{Li}^{1,2}$
}

'Department of General Surgery, Qilu Hospital, Shandong University, Jinan, People's Republic of China; ${ }^{2}$ Department of Hepatobiliary Surgery, The Second Hospital of Shandong University, Jinan, People's Republic of China

*These authors contributed equally to this work
Correspondence: Tao Li

Tel +86-53I-82I6665I

Email litao7706@I63.com

\begin{abstract}
The Rab GTPase family contains almost 70 genes in the human genome and acts as the key regulator of intracellular membrane trafficking in human cells. The dysregulation of Rab GTPase has been shown to be associated with multiple human diseases, ranging from neurodegeneration, and infection to cancer. Rab GTPases not only play important roles in genome replication, morphogenesis and the release of hepatitis B virus (HBV) or hepatitis $\mathrm{C}$ virus (HCV), but also contribute to hepatitis-related hepatocarcinogenesis and hepatocellular carcinoma (HCC) progression. The alteration of Rab GTPase expression in HCC plays an important role in tumour cell proliferation, invasion and migration. Notably, the expression of Rab genes can be regulated by some noncoding RNAs, such as miRNAs and circRNAs. Thus, Rab GTPases can serve as promising rational and therapeutic targets for HCC treatments. In this review, we summarized recent advancements in this field focusing on Rab GTPases in HCC.
\end{abstract}

Keywords: Rab GTPase, membrane trafficking, hepatocellular carcinoma, therapeutic target

\section{Background}

Hepatocellular carcinoma (HCC) is the most common type of primary liver cancer and the fifth most common neoplasm. It accounts for approximately $8 \%$ of all cancer-related deaths and more than 800,000 new cases and deaths globally each year. ${ }^{1}$ HCC has been recognized as the leading cause of death among patients with cirrhosis. In recent decades, there has been great progress in the diagnosis and treatment of $\mathrm{HCC}$, but the prognosis of HCC patients is still unsatisfactory. ${ }^{2,3}$ The prevention and treatment of HCC are still some of the most serious public health problems in the world. It is urgent to look for early diagnostic markers and therapeutic targets, especially those that can be exploited to inhibit angiogenesis and alter the oxygen-deficient environment, to improve HCC patient prognosis and quality of life. ${ }^{4-6}$

Intracellular membrane trafficking is crucial for regulating membrane transport and dynamics for a variety of membranous organelles in eukaryotic cells. Rab GTPases, which include almost 70 highly evolutionarily conserved genes in the human genome, play important roles in intracellular membrane trafficking. They are localized to different membrane compartments and exert their function by recruiting effector molecules to control the specificity and directionality of membrane protein trafficking, vesicular transport, membrane targeting and fusion. ${ }^{7}$

Notably, Rab GTPases can be involved in tumour cell migration, invasion, proliferation, stromal cell communication, and drug resistance. ${ }^{8}$ The activity of Rab GTPases can be regulated by guanine nucleotide exchange factors (GEFs) or GTPaseactivating proteins (GAPs) through the interconversion of the active GTP-bound form 
and the inactive GDP-bound form. ${ }^{9}$ Intracellular transport dysfunction induced by aberrant Rab GTPases in cancers has attracted growing concern. Our review delineates recent advances regarding the molecular mechanism of Rab GTPases and the roles of these GTPases in hepatocarcinogenesis and HCC progression.

\section{The Molecular Mechanism of Rab GTPases as Switches in Membrane Trafficking}

As they are molecular switches, Rab GTPases can be active when GTP is bound and inactive when GDP is bound. The oscillation between these two distinct conformations is tightly controlled by specific proteins, such as guanine nucleotide exchange factors (GEFs) and GAPs. ${ }^{10}$ In detail, GEFs facilitate GTP binding with GTPases and contribute to the conversion to an active state, whereas the inactive form is coordinated by GAPs, which catalyse
GTP hydrolysis. ${ }^{11}$ To anchor to correct internal membranes, Rab GTPases need to be prenylated at their C-termini. ${ }^{12}$ As a Rab-specific effector, guanine nucleotide dissociation inhibitor (GDI) has a high affinity for GDPbound Rab proteins and plays an important role in mediating the dissociation of membrane-bound Rab GTPases from membranes to the cytosol (Figure 1). ${ }^{13}$

The role of GTP-bound Rab protein in facilitating specific and accurate transport depends on biological interactions with multiple effectors, including tethering factors, motor linkers, Rab regulators, and sorting adaptors. ${ }^{14}$ These effectors coordinate with Rab GTPases to complete the consecutive processes of vesicle trafficking along endocytic and exocytic paths, such as receptor cargo selection, vesicle formation and movement along the cytoskeleton, accurate docking and further fusion with their target membrane compartments. For instance, Rab coupling protein (FIP1C) and Rab11 family interacting protein 2 (Rab11-FIP2) are the effectors of RAb11; Rab11-FIP2 was found to regulate endosome

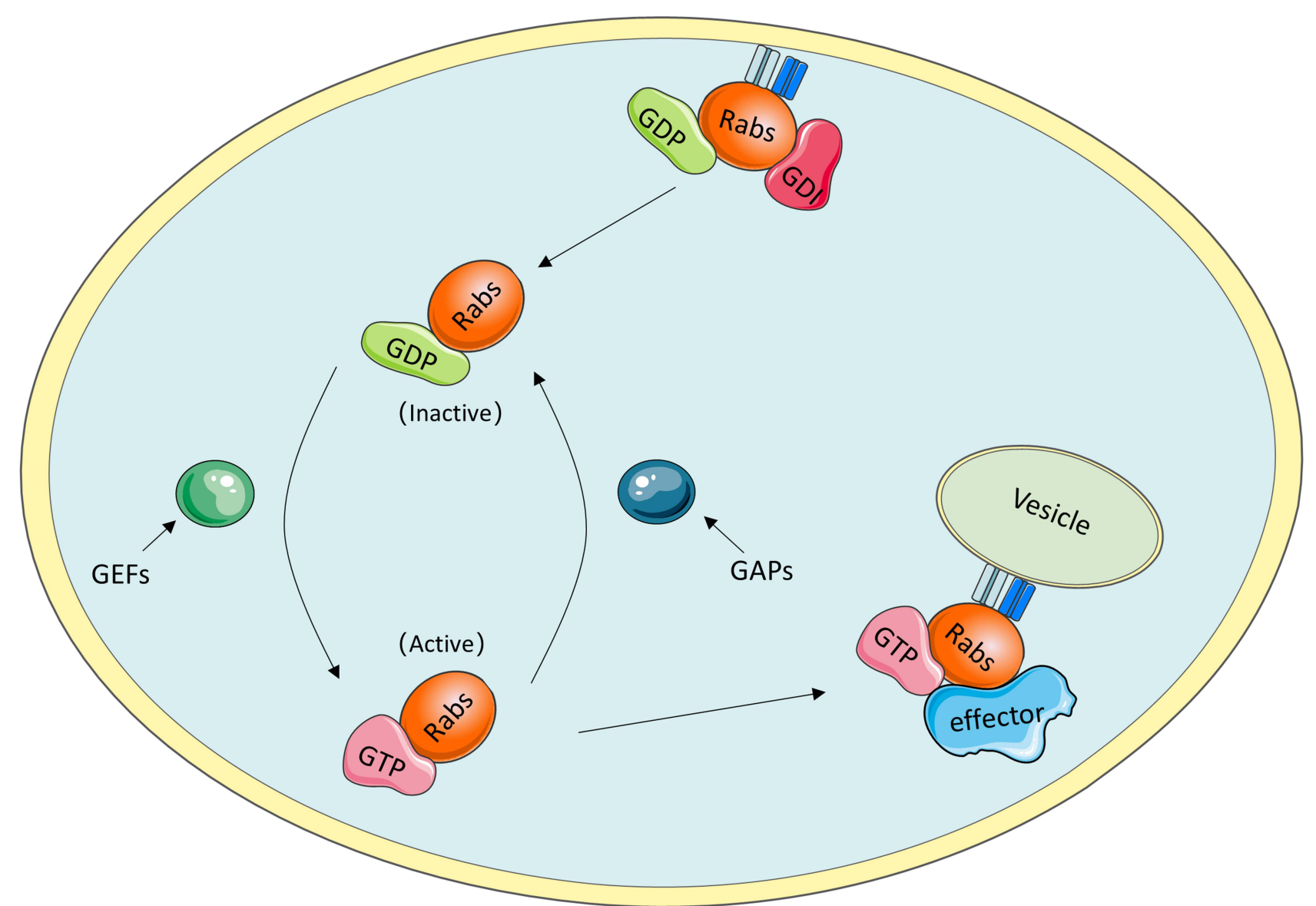

Figure I Rab GTPases cycle. i) GDI binding with GDP-bound Rab proteins mediates the dissociation of membrane-bound Rab GTPases from membranes to the cytosol. ii) GEFs facilitate the conversion to active GTP-bound Rab proteins, whereas the transition to inactive GDP-bound Rab proteins is coordinated by GAPs through catalyzing GTP hydrolysis. iii) GTP-bound Rab proteins interact with multiple effectors to execute specific functions. 
trafficking and promotes cellular motility in nasopharyngeal carcinoma, and targeted deletion of FIp1C led to accelerated tumour onset in the context of Erbb2-positive breast cancer. $^{15,16}$

The localization of Rabs to their appropriate intracellular membranes after prenylation requires Rab escort protein (REP), which includes two components: choroideremia (CHM) and choroideremia-like (CHML). ${ }^{17}$ The expression of CHML is increased in HCC tissues and is associated with prognosis in HCC patients. A mechanistic study revealed that CHML facilitates constant Rab14 recycling by escorting Rab14 to the membrane and then promoting HCC migration, invasion and metastasis. Thus, the CHML-Rab14 axis has been established as a potential promoter of HCC progression and may be employed as a promising therapeutic target for HCC treatment. ${ }^{18,19}$

\section{Effects of Rab GTPase Dysregulation}

Due to their regulation of the indispensable process of membrane trafficking, Rab proteins are crucial for the maintenance of cell homeostasis and concrete cellular functions. The dysregulation or mutation of Rab proteins or their related effectors not only affects the process of membrane transport but also causes disorders of multiple cellular processes, such as cell division, migration, apoptosis and survival. $^{20}$ Several genetic diseases, such as diabetes, immune deficiency and cancer, are driven by mutations in Rab GTPases, and their interacting proteins have been identified. $^{21}$ The abnormal function of Rab GTPases is associated with a variety of HCC pathophysiologies (Figure 2).

Autophagy plays a crucial role in resistance to nutrient deficiency and the removal of intracellular misfolded proteins and damaged senescent organelles. ${ }^{22}$ As a kind of self-protective mechanism, autophagy is indispensable for the maintenance of cellular proteostasis and homeostasis. Dysregulation of autophagy can lead to the development of a variety of diseases, including cancers. ${ }^{23}$ As the main regulators of autophagy, Rab GTPases can link autophagic degradation with the intracellular membrane dynamics network and facilitate tumorigenesis and cancer development. $^{24,25}$ Dysregulation of autophagy has been noted in the pathogenesis of HCC. ${ }^{26}$ Rab GTPases can affect the process of autophagy and play a driving role in the occurrence and progression and even the metastasis of HCC. As an LRRK2 substrate, Rab GTPases can not only

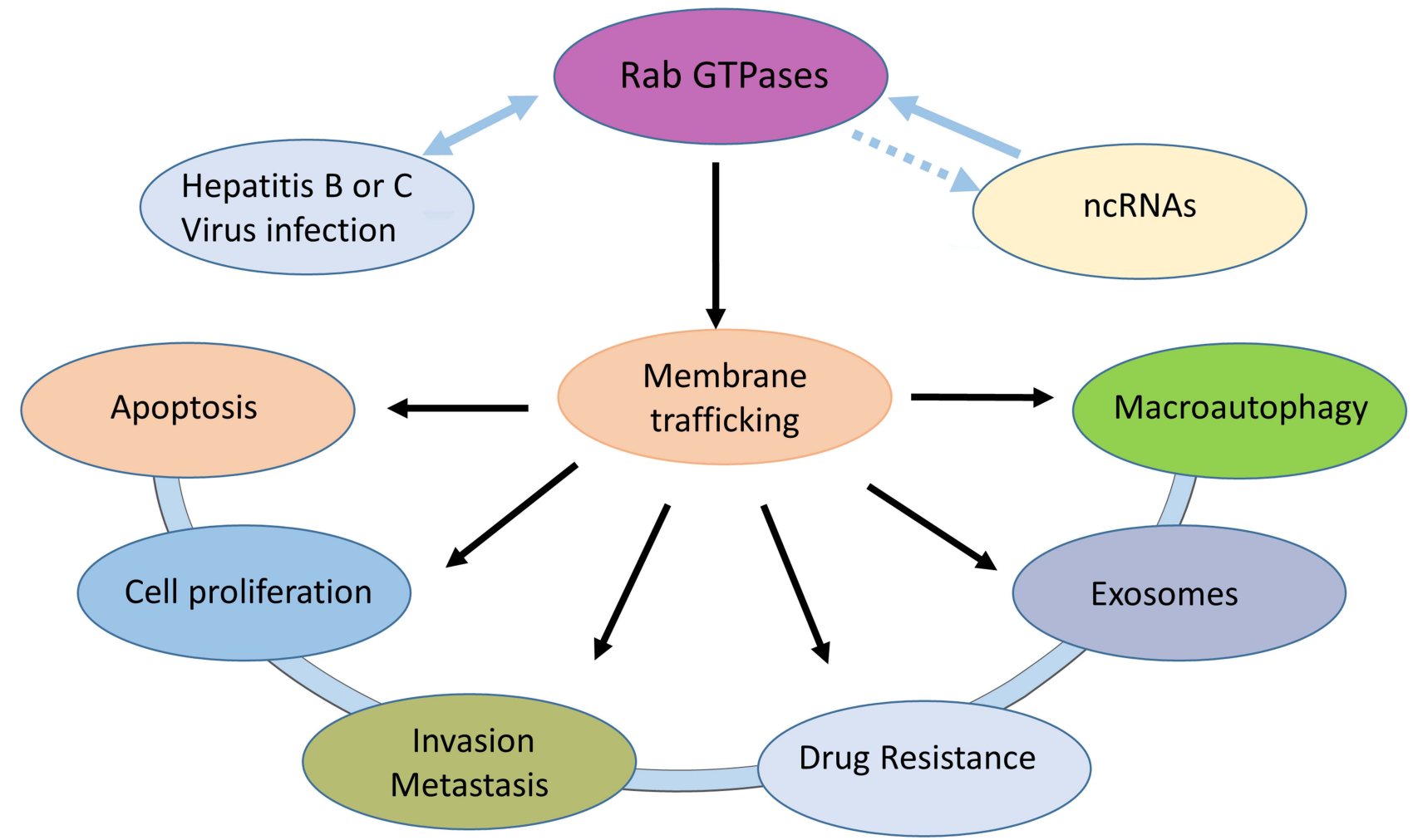

Figure 2 Rab GTPases can affect cell proliferation, apoptosis, invasion, metastasis, exosomes and autophagy by mediating membrane transport. Hepatitis B or C virus can interact with Rab GTPases. Some noncoding RNAs can regulate Rab GTPases expression in HCC. 
indirectly regulate phagocytosis, cell signal transduction and autophagy in immune cells, ${ }^{27}$ but they also serve as regulators of cilia formation and immune synaptic assembly. ${ }^{28}$

As mediators of intercellular communication that carry hereditary material and molecular information, exosomes have been demonstrated to be involved in a variety of processes, including immune responses, cytokinesis, neurite outgrowth, blood coagulation, angiogenesis, and cancer metastatic dissemination and migration, based on their unique structure and distinct origins. ${ }^{29-31}$ Rab GTPases have been identified to critically regulate exosome biogenesis and secretion. ${ }^{32}$ Recent studies have found that knockdown of Rab27a inhibits exosome secretion and thus promotes the migration and invasion of parental MHCC97H cells. Further study showed that exosomes extracted from MHCC97H cells can promote the process of epithelial-mesenchymal transition (EMT) by activating the MAPK/ERK signalling pathway. ${ }^{33}$

\section{Relationship Between Rab GTPases and Hepatitis B Virus (HBV) or Hepatitis C Virus (HCV)}

HCC appears frequently in the context of cirrhosis and HBV or HCV infections. ${ }^{34}$ Over $50 \%$ of HCC cases worldwide are attributed to $\mathrm{HBV}$, which makes $\mathrm{HBV}$ one of the most common carcinogens. HBV can promote hepatocyte transformation, HCC development and progression through a series of driving stimuli, including DNA damage, chronic inflammation, senescence, epigenetic modifications, telomerase reactivation and chromosomal instability. Recent studies have revealed that HBV can promote hepatocarcinogenesis and HCC progression by regulating Rab GTPase expression and activity. ${ }^{35} \mathrm{HBV}$ can promote $\mathrm{HCC}$ cell proliferation and migration by downregulating the expression of miR-101-3p targeting Rab5a. ${ }^{36}$ Emerging evidence has revealed that HBx can induce the upregulation of oncogenic Rab18 in HCC and then enhance hepatocarcinogenesis, contributing to the abnormal lipogenesis and hyperproliferation in HBVassociated hepatomas. ${ }^{37}$

Rab GTPases are also involved in the processes of infection, genome replication, morphogenesis and $\mathrm{HBV}$ or $\mathrm{HCV}$ release. After endocytosis, HBV travels through a complex network of endocytic pathways to the nucleus and begins to replicate. Endosomal vesicles may play a key role in infection, providing the right environment for viral uncoating and nucleocapsid release. Rab GTPases play an important role in vesicular transport, which is involved in different stages of the viral life cycle. First, HBV requires Rab5- and Rab7dependent transport through the endosomal compartment to initiate a successful infection. ${ }^{38}$ On the other hand, HBV can suppress autophagosome maturation and escape autophagic degradation via the negative regulation of Rab7 expression. ${ }^{39}$ In addition, Rab33B is necessary for the reproduction of HBV and controls HBV assembly by regulating core membrane binding and nucleocapsid processing. ${ }^{40}$

Membrane transport is also involved in the life cycle of the hepatitis $\mathrm{C}$ virus (HCV). The Rab protein is an important host factor for HCV RNA replication. First, Rab13 regulates the endocytic cycle of tight junction-associated proteins, whose downregulation inhibits $\mathrm{HCV}$ infection. Therefore, Rab13 plays an important role in the entry of $\mathrm{HCV}^{41}$ Second, the formation of the HCV replication complex requires RAB-associated proteins such as RAB5 and RAB7. ${ }^{42}$ In addition, Rab5, Rab7, Rab18 and Rab27a are important for HCV genome replication. Rab1b, Rab3d, Rab7, Rab9 and Rab27a are involved in the release of $\mathrm{HCV}^{43} \mathrm{RAB} 18$ and $\mathrm{RAB} 32$ are required for the viral assembly of $\mathrm{HCV}^{44-46}$

\section{Relationship Between Rab GTPases and HCC}

As Rab-mediated membrane transport affects many cellular functions, Rab GTPases can also indirectly control several cell signalling pathways involved in cell division, migration, apoptosis, survival, tumorigenesis, and cancer progression. ${ }^{20}$ Given their pivotal roles in membrane trafficking and the maintenance of cellular homeostasis, Rabs and Rab-associated factors are implicated in a myriad of human diseases, particularly HCC. Previous studies have demonstrated that several Rab GTPases are involved in hepatocarcinogenesis and HCC progression (Table 1). Most aberrant Rab GTPases, such as RAB11A, are overexpressed in HCC and can activate tumour growth, survival, invasion and metastasis via various signalling pathways, such as the phosphoinositide 3-kinase (PI3K) pathway and the mitogen-activated protein kinase (MAPK) pathway. ${ }^{47}$

Rab GTPases are rarely downregulated in HCC tissues and function as tumour suppressors. Studies reveals that Rab17 plays an unexpected role as a tumour suppressor whose overexpression in HCC cells attenuates tumorigenic properties, including cell proliferation, migration and invasion, by deactivating the Erk pathway. ${ }^{48,49}$ RAB43 is 
Table I Rab GTPases Functions and Prognostic Implications in HCC

\begin{tabular}{|l|l|l|}
\hline Gene Name & Function & Prognostic Implication \\
\hline Rab5a & Promoting cell growth, differentiation, apoptosis and angiogenesis & Tumour promoter factor \\
Rabl0 & Regulating cell survival and proliferation & Tumour promoter factor \\
RabIla & Promoting migration and invasion & Tumour promoter factor \\
RabI7 & Inhibiting migration and invasion and promoting GI arrest & Tumour suppressor factor \\
Rab18 & Enhancing hepatocarcinogenesis and cell proliferation & Tumour promoter factor \\
Rab25 & Promoting migration and invasion & Tumour promoter factor \\
Rab27a & Inhibiting migration and invasion & Tumour suppressor factor \\
Rab3I & Promoting migration and invasion & Tumour promoter factor \\
Rab40b & Promoting migration and invasion & Tumour promoter factor \\
Rab43 & Inhibiting cell proliferation and EMT & Tumour suppressor factor \\
\hline
\end{tabular}

another downregulated Rab GTPase in HCC tissues. Overexpression of RAB43 can inhibit the proliferation of HCC cells, while knockdown of RAB43 can promote the EMT process in $\mathrm{HCC}^{50}$

\section{Rab GTPases Regulate HCC Cell Proliferation, Apoptosis, Invasion and Metastasis}

Rab GTPases have been reported to regulate HCC cell proliferation and apoptosis. First, high RAB10 levels in HCC cells are correlated with a poor prognosis in HCC patients. In vitro and in vivo studies have shown that RAB10 plays an oncogenic role in regulating cell survival and proliferation through multiple oncogenic, cell stress and apoptosis pathways in HCC. ${ }^{51}$ Rabl3 is also a valuable marker for the prediction of HCC progression and a prognostic indicator for $\mathrm{HCC}$ patient prognosis. Overexpression of Rabl3 can significantly enhance the proliferation and growth of hepatoma cells. ${ }^{52,53}$ Rab31 is another Rab GTPase that intensively inhibits apoptosis and accelerates growth by activating the $\mathrm{PI} 3 \mathrm{~K} / \mathrm{AKT}$ pathway and increasing the $\mathrm{Bcl}-2 / \mathrm{BAX}$ ratio in $\mathrm{HCC} .^{54,55}$

Increasing evidence has revealed that there are some Rab GTPases that not only promote HCC proliferation, but also promote HCC invasion and metastasis. As a highly expressed Rab GTPase in HCC tissues that is associated with poor clinical prognosis, Rab11a can promote HCC cell proliferation, migration and invasion and inhibit apoptosis by activating the $\mathrm{PI} 3 \mathrm{~K} / \mathrm{AKT}$ pathway and regulating matrix metalloproteinase 2 (MMP2) expression. ${ }^{56}$ As a newly identified mammalian target of rapamycin complex 1 (mTORC1) activator, Rab1A is reported to be frequently overexpressed in HCC samples and can promote $\mathrm{HCC}$ growth and metastasis and increase rapamycin treatment sensitivity by enhancing hyperactive amino acidmTORC1 signalling in vitro and in vivo. ${ }^{57,58}$

Rab5a, Rab25 and Rab40 are also upregulated in HCC tissues compared with paratumour tissues. The level of Rab5a in HCC samples has been associated with tumour-nodemetastasis (TNM) stage and lymph node metastasis. Mechanistically, Rab5a promotes cell proliferation, invasion and migration by activating the EGF receptor and FAK signalling pathways. ${ }^{59}$ Rab25 promotes HCC cell malignant proliferation and invasion by activating the AKT and Wnt signalling pathways. ${ }^{60}$ Overexpression of Rab40b upregulates cyclin D1, cyclin E1 and MMP2 to promote HCC proliferation and metastasis through the PI3K/AKT signalling pathway. ${ }^{61}$

Rab27a is another important and unique Rab GTPase that regulates the process of $\mathrm{HCC}$ cell exosome secretion. The knockdown of Rab27a can inhibit MHCC97H-derived exosome secretion, which then promotes the migration, chemotaxis and invasion of MHCC97H cells by regulating EMT via the MAPK/ERK signalling pathway. ${ }^{62}$ Rab34 can also augment cell migration and invasion properties by regulating the G1 phase of the cell cycle and promoting the EMT process. $^{63,64}$

\section{Rab Family Genes are Regulated by Noncoding RNAs}

Aberrant expression of noncoding RNAs in cancer samples has been reported to be correlated with clinicopathological parameters and prognosis in patients with malignancies, including HCC. As expected, some altered noncoding RNAs, including miRNAs, in HCC can regulate cell proliferation, invasion, and metastasis and influence HCC patient prognosis by targeting Rab family genes (Table 2). ${ }^{65}$ For example, miR-101-3p, a downregulated miRNA in HCC tissues, can regulate $\mathrm{HCC}$ cell growth and 
Table 2 Rab GTPases-Associated Noncoding RNAs in HCC

\begin{tabular}{|l|l|l|l|l|}
\hline Gene Name & \multicolumn{1}{|c|}{ Non-Coding RNA } & Pathway & \multicolumn{1}{|c|}{ Function in HCC } & Reference \\
\hline Rab5a & MiR-10I-3p & NA & Cell growth and migration & [66] \\
\hline RabI(Rabla) & $\begin{array}{l}\text { MiR-223 } \\
\text { Circ-UGGT2 } \\
\text { MiR-5226B-5P }\end{array}$ & mTOR & Cell proliferation, migration, invasion and apoptosis & {$[67,72]$} \\
\hline Rablb & MiR-54I & NA & Positive modulator of autophagy & [68] \\
\hline RabI8 & MiR-145-5P & NA & Promote proliferation and migration and inhibit the apoptosis & [69] \\
\hline Rab23 & MiR-429 & EMT & Promotes cell growth and invasion and represses apoptosis & [70] \\
\hline Rab24 & MiR-615-5p & Rab-RAS & Retarding cell cycling processes and accelerating apoptosis & [7I] \\
\hline Rab35 & Long non-coding RNA HOTAIP & mTOR & Promotes exosome secretion & [73] \\
\hline
\end{tabular}

migration by targeting the Rab5A gene. ${ }^{66}$ MiR-223, a well-identified downregulated miRNA in HCC samples, modulates cell proliferation through regulation of cell apoptosis in a Rab1-mTORC1-dependent manner. ${ }^{67}$ As a miRNA associated with malignant clinicopathologic phenotypes and prognosis and sorafenib treatment sensitivity of HCC patients, miR-541 inhibits the malignant properties and autophagy of HCC cells both in vitro and in vivo by partly downregulating the expression of Rab1B which serves as an oncogene and important modulator of autophagy in HCC. ${ }^{68}$ Wang et al also reported that miR$145-5 \mathrm{p}$ acts as a new tumour suppressor by specifically targeting RAB18 whose ectopic expression can promote proliferation and migration and inhibit the apoptosis of HCC cells. ${ }^{69}$ More interestingly, miR-429 decreases the migratory ability and reverses the process of EMT (thus promoting mesenchymal-epithelial transition [MET]) in HCC by targeting RAB23. ${ }^{70}$ In addition, miR-615-5p inhibits HCC cell malignant growth abilities, such as cell motility and metastasis, by retarding cell cycling processes and accelerating apoptosis with a direct inhibitory effect on Rab24. ${ }^{71}$

The expression of Rab family genes can be regulated by other noncoding RNAs, such as circRNAs and long noncoding RNAs. For example, the silencing of circUGGT2 inhibits HCC growth in vivo and restrains the proliferation, colony formation, cell cycle progression, migration, and invasion of HCC cells in vitro by competitively binding to miR-526b-5p which targets RAB1A. ${ }^{72}$ Long noncoding RNA HOTAIR promotes exosome secretion by regulating $\mathrm{RAB} 35$ expression and localization in HCC. $^{73}$ Long noncoding RNA HULC promotes HCC cell growth and invasion and represses apoptosis by regulating the miR-372-3p/RAB23 axis. $^{74}$

\section{Conclusion}

Considering the prominent role in endomembrane dynamics, Rab GTPases indeed occupy a dominant position not only in the maintenance of proteostasis and homeostasis but also in cell type-specific functions. It is not surprising that altered expression of Rab GTPases correlates with various human diseases, particularly cancer. Alterations in expression rather than mutations of Rab genes play master regulatory roles in HCC pathological processes, including cell motility, proliferation, metastasis and survival, via the vital impact on vesicle trafficking, signalling cascades, and receptor recycling. Intriguingly, Rab GTPases play bidirectional roles in the physiology of tumour processes. Most Rab GTPases are viewed as oncogenic drivers and are associated with tumorigenesis, progression and metastasis, and the underlying molecular mechanisms are not well recognized and need further research.

A new type of tumour biomarker based on abnormal alterations of Rab GTPases in HCC patients may be used for the early diagnosis of HCC. The expression levels of numerous Rab GTPase have been verified to be closely associated with tumour progression and HCC patient prognosis and thus probably serve as putative prognostic indicators. In addition, identification of the physiologic and pathophysiologic characteristics of Rab GTPases may offer innovative perspectives to exploit effective small molecule inhibitors and therapeutic strategies related to Rab GTPases for HCC treatment. 


\section{Abbreviations}

$\mathrm{HCC}$, hepatocellular carcinoma; GEF, guanine nucleotide exchange factors; GAP, GTPase-activating proteins; GDI, guanine nucleotide dissociation inhibitor; $\mathrm{CHML}$, choroideremia-like; HBV, hepatitis $\mathrm{B}$ virus; $\mathrm{HCV}$, hepatitis $\mathrm{C}$ virus; MAPK, mitogen-activated protein kinase; EMT, epithelial-mesenchymal transformation; MMP2, matrix metalloproteinase 2 .

\section{Data Sharing Statement}

All data are included in the article.

\section{Acknowledgments}

We thank all of the original studies for sharing their data.

\section{Funding}

This study was supported by the Taishan Scholars Program for Young Expert of Shandong Province (tsqn20161064), the National Natural Science Foundation of China (81874178, 82172647, 82073200), Independent Cultivation of Innovative Team from Universities in Jinan (Grant No.2020GXRC023) and China Postdoctoral Science Foundation (2020M682192).

\section{Disclosure}

The authors report no conflicts of interest in this work.

\section{References}

1. Bray F, Ferlay J, Soerjomataram I, Siegel RL, Torre LA, Jemal A. Global cancer statistics 2018: GLOBOCAN estimates of incidence and mortality worldwide for 36 cancers in 185 countries. CA Cancer J Clin. 2018;68(6):394-424. doi:10.3322/caac.21492

2. Dong ZR, Zhou W, Sun D, et al. Role of the E3 ubiquitin ligase TRIM4 in predicting the prognosis of hepatocellular carcinoma. $J$ Cancer. 2020;11(14):4007-4014. doi:10.7150/jca.37164

3. Balogh J, Victor D 3rd, Asham EH, et al. Hepatocellular carcinoma: a review. J Hepatocell Carcinoma. 2016;3:41-53. doi:10.2147/JHC. S61146

4. Dong ZR, Sun D, Yang YF, et al. TMPRSS4 drives angiogenesis in hepatocellular carcinoma by promoting HB-EGF expression and proteolytic cleavage. Hepatology. 2020;72(3):923-939. doi:10.1002/ hep. 31076

5. Dong XF, Liu TQ, Zhi XT, et al. COX-2/PGE2 axis regulates HIF2 $\alpha$ activity to promote hepatocellular carcinoma hypoxic response and reduce the sensitivity of sorafenib treatment. Clin Cancer Res. 2018;24(13):3204-3216. doi:10.1158/1078-0432.CCR-17-2725

6. Yim SY, Lee JS. An overview of the genomic characterization of hepatocellular carcinoma. $J$ Hepatocell Carcinoma. 2021;8:1077-1088. doi:10.2147/JHC.S270533

7. Stenmark H. Rab GTPases as coordinators of vesicle traffic. Nat Rev Mol Cell Biol. 2009;10(8):513-525. doi:10.1038/nrm2728

8. Recchi C, Seabra MC. Novel functions for Rab GTPases in multiple aspects of tumour progression. Biochem Soc Trans. 2012;40 (6):1398-1403. doi:10.1042/BST20120199
9. Müller MP, Goody RS. Molecular control of Rab activity by GEFs, GAPs and GDI. Small GTPases. 2018;9(1-2):5-21. doi:10.1080/ 21541248.2016.1276999

10. Pylypenko O, Hammich H, Yu IM, Houdusse A. Rab GTPases and their interacting protein partners: structural insights into Rab functional diversity. Small GTPases. 2018;9(1-2):22-48. doi:10.1080/ 21541248.2017.1336191

11. Barr F, Lambright DG. Rab GEFs and GAPs. Curr Opin Cell Biol. 2010;22(4):461-470. doi:10.1016/j.ceb.2010.04.007

12. Zhang FL, Casey PJ. Protein prenylation: molecular mechanisms and functional consequences. Аnnu Rev Biochem. 1996;65:241-269. doi:10.1146/annurev.bi.65.070196.001325

13. Wu YW, Tan KT, Waldmann H, Goody RS, Alexandrov K. Interaction analysis of prenylated Rab GTPase with Rab escort protein and GDP dissociation inhibitor explains the need for both regulators. Proc Natl Acad Sci U S A. 2007;104(30):12294-12299. doi:10.1073/pnas.0701817104

14. Gillingham AK, Sinka R, Torres IL, Lilley KS, Munro S. Toward a comprehensive map of the effectors of rab GTPases. Dev Cell. 2014;31(3):358-373. doi:10.1016/j.devcel.2014.10.007

15. Boulay PL, Mitchell L, Turpin J, et al. Rab11-FIP1C is a critical negative regulator in ErbB2-mediated mammary tumor progression. Cancer Res. 2016;76(9):2662-2674. doi:10.1158/0008-5472.CAN15-2782

16. Feng G, Qin L, Liao Z, et al. Knockdown Rab11-FIP2 inhibits migration and invasion of nasopharyngeal carcinoma via suppressing Rho GTPase signaling. J Cell Biochem. 2020;121(2):1072-1086. doi:10.1002/jcb.29344

17. Yun UJ, Sung JY, Park SY, et al. Oncogenic role of rab escort protein 1 through EGFR and STAT3 pathway. Cell Death Dis. 2017;8(2): e2621. doi:10.1038/cddis.2017.50

18. Chen TW, Yin FF, Yuan YM, et al. CHML promotes liver cancer metastasis by facilitating Rab14 recycle. Nat Commun. 2019;10 (1):2510. doi:10.1038/s41467-019-10364-0

19. Zhang W, Cao L, Liu X, et al. High expression of CHML predicts poor prognosis of multiple myeloma. J Cancer. 2019;10 (24):6048-6056. doi:10.7150/jca.34465

20. Pellinen T, Arjonen A, Vuoriluoto K, Kallio K, Fransen JA, Ivaska J. Small GTPase Rab21 regulates cell adhesion and controls endosomal traffic of beta1-integrins. $J$ Cell Biol. 2006;173(5):767-780. doi:10.1083/jcb.200509019

21. Mitra S, Cheng KW, Mills GB. Rab GTPases implicated in inherited and acquired disorders. Semin Cell Dev Biol. 2011;22(1):57-68. doi:10.1016/j.semcdb.2010.12.005

22. White E, Mehnert JM, Chan CS. Autophagy, metabolism, and cancer. Clin Cancer Res. 2015;21(22):5037-5046. doi:10.1158/1078-0432. CCR-15-0490

23. Akkoç Y, Gözüaçık D. Autophagy and liver cancer. Turk J Gastroenterol. 2018;29(3):270-282. doi:10.5152/tjg.2018.150318

24. Takats S, Boda A, Csizmadia T, Juhasz G. Small GTPases controlling autophagy-related membrane traffic in yeast and metazoans. Small GTPases. 2018;9(6):465-471. doi:10.1080/21541248.2016.1258444

25. Lu Q, Wang PS, Yang L. Golgi-associated Rab GTPases implicated in autophagy. Cell Biosci. 2021;11(1):35. doi:10.1186/s13578-02100543-2

26. Zhu Y, Wang R, Chen W, Chen Q, Zhou J. Construction of a prognosis-predicting model based on autophagy-related genes for hepatocellular carcinoma (HCC) patients. Aging. 2020;12 (14):14582-14592. doi:10.18632/aging. 103507

27. Wallings RL, Tansey MG. LRRK2 regulation of immune-pathways and inflammatory disease. Biochem Soc Trans. 2019;47 (6):1581-1595. doi:10.1042/BST20180463

28. Patrussi L, Baldari CT. The Rab GTPase Rab8 as a shared regulator of ciliogenesis and immune synapse assembly: from a conserved pathway to diverse cellular structures. Small GTPases. 2016;7 (1):16-20. doi:10.1080/21541248.2015.1111852 
29. Peinado H, Alec`ković M, Lavotshkin S, et al. Corrigendum: melanoma exosomes educate bone marrow progenitor cells toward a pro-metastatic phenotype through MET. Nat Med. 2016;22 (12):1502. doi:10.1038/nm1216-1502b

30. Muralidharan-Chari V, Clancy J, Plou C, et al. ARF6-regulated shedding of tumor cell-derived plasma membrane microvesicles. Curr Biol. 2009;19(22):1875-1885. doi:10.1016/j.cub.2009. 09.059

31. Yu X, Prekeris R, Gould GW. Role of endosomal Rab GTPases in cytokinesis. Eur J Cell Biol. 2007;86(1):25-35. doi:10.1016/j. ejcb.2006.10.002

32. Blanc L, Vidal M. New insights into the function of Rab GTPases in the context of exosomal secretion. Small GTPases. 2017;9(12):95-106. doi:10.1080/21541248.2016.1264352

33. Chen L, Guo P, He Y, et al. HCC-derived exosomes elicit HCC progression and recurrence by epithelial-mesenchymal transition through MAPK/ERK signalling pathway. Cell Death Dis. 2018;9 (5):513. doi:10.1038/s41419-018-0534-9

34. Yang JD, Hainaut P, Gores GJ, Amadou A, Plymoth A, Roberts LR. A global view of hepatocellular carcinoma: trends, risk, prevention and management. Nat Rev Gastroenterol Hepatol. 2019;16 (10):589-604. doi:10.1038/s41575-019-0186-y

35. $\mathrm{Ng} \mathrm{SA}$, Lee C. Hepatitis B virus X gene and hepatocarcinogenesis. J Gastroenterol. 2011;46(8):974-990. doi:10.1007/s00535-011-0415-9

36. Sheng Y, Li J, Zou C, et al. Downregulation of miR-101-3p by hepatitis $\mathrm{B}$ virus promotes proliferation and migration of hepatocellular carcinoma cells by targeting Rab5a. Arch Virol. 2014;159 (9):2397-2410. doi:10.1007/s00705-014-2084-5

37. You X, Liu F, Zhang T, Li Y, Ye L, Zhang X. Hepatitis B virus $\mathrm{X}$ protein upregulates oncogene Rab18 to result in the dysregulation of lipogenesis and proliferation of hepatoma cells. Carcinogenesis. 2013;34(7):1644-1652. doi:10.1093/carcin/bgt089

38. Macovei A, Petrareanu C, Lazar C, Florian P, Branza-Nichita N. Regulation of hepatitis B virus infection by Rab5, Rab7, and the endolysosomal compartment. J Virol. 2013;87(11):6415-6427. doi:10.1128/JVI.00393-13

39. Zhou T, Jin M, Ding Y, et al. Hepatitis B virus dampens autophagy maturation via negative regulation of Rab7 expression. Biosci Trends. 2016;10(4):244-250. doi:10.5582/bst.2016.01049

40. Bartusch C, Döring T, Prange R. Rab33B controls hepatitis B virus assembly by regulating core membrane association and nucleocapsid processing. Viruses. 2017;9(6):157. doi:10.3390/v9060157

41. Takeda M, Ikeda M, Satoh S, Dansako H, Wakita T, Kato N. Rab13 is involved in the entry step of hepatitis C virus infection. Acta Med Okayama. 2016;70(2):111-118.

42. Manna D, Aligo J, Xu C, et al. Endocytic Rab proteins are required for hepatitis $\mathrm{C}$ virus replication complex formation. Virology. 2010;398(1):21-37. doi:10.1016/j.virol.2009.11.034

43. Elgner F, Hildt E, Bender D. Relevance of Rab proteins for the life cycle of hepatitis C virus. Front Cell Dev Biol. 2018;6:166. doi:10.3389/fcell.2018.00166

44. Dansako H, Hiramoto H, Ikeda M, Wakita T, Kato N. Rab18 is required for viral assembly of hepatitis $\mathrm{C}$ virus through trafficking of the core protein to lipid droplets. Virology. 2014;462463:166-174. doi:10.1016/j.virol.2014.05.017

45. Salloum S, Wang H, Ferguson C, Parton RG, Tai AW. Rab18 binds to hepatitis C virus NS5A and promotes interaction between sites of viral replication and lipid droplets. PLoS Pathog. 2013;9(8): e1003513. doi:10.1371/journal.ppat.1003513

46. Pham TM, Tran SC, Lim YS, Hwang SB. Hepatitis C virus-induced Rab32 aggregation and its implications for virion assembly. $J$ Virol. 2017;91(3):e01662-16. doi:10.1128/JVI.01662-16

47. Tsuruta F, Masuyama N, Gotoh Y. The phosphatidylinositol 3-kinase (PI3K)-Akt pathway suppresses Bax translocation to mitochondria. $J$ Biol Chem. 2002;277(16):14040-14047. doi:10.1074/jbc. M108975200
48. Von Thun A, Birtwistle M, Kalna G, et al. ERK2 drives tumour cell migration in three-dimensional microenvironments by suppressing expression of Rab17 and liprin-beta2. J Cell Sci. 2012;125(Pt 6):1465-1477.

49. Wang K, Mao Z, Liu L, et al. Rab17 inhibits the tumourigenic properties of hepatocellular carcinomas via the Erk pathway. Tumour Biol. 2015;36(8):5815-5824. doi:10.1007/s13277-015-3251-3

50. Guo L, Gao R, Gan J, et al. Downregulation of TNFRSF19 and RAB43 by a novel miRNA, miR-HCC3, promotes proliferation and epithelial-mesenchymal transition in hepatocellular carcinoma cells. Biochem Biophys Res Commun. 2020;525(2):425-432. doi:10.1016/j. bbrc.2020.02.105

51. Wang W, Jia WD, Hu B, Pan YY. RAB10 overexpression promotes tumor growth and indicates poor prognosis of hepatocellular carcinoma. Oncotarget. 2017;8(16):26434-26447. doi:10.18632/ oncotarget. 15507

52. Pan Y, Liu Z, Feng Z, et al. The overexpression of Rabl3 is associated with pathogenesis and clinicopathologic variables in hepatocellular carcinoma. Tumour Biol. 2017;39(4):1010428317696230. doi: $10.1177 / 1010428317696230$

53. An J, Liu Z, Liang Q, et al. Overexpression of Rabl3 and Cullin7 is associated with pathogenesis and poor prognosis in hepatocellular carcinoma. Hum Pathol. 2017;67:146-151. doi:10.1016/j. humpath.2017.07.008

54. Sui Y, Zheng X, Zhao D. Rab31 promoted hepatocellular carcinoma (HCC) progression via inhibition of cell apoptosis induced by PI3K/ AKT/Bcl-2/BAX pathway. Tumour Biol. 2015;36(11):8661-8670. doi:10.1007/s13277-015-3626-5

55. Chua CE, Tang BL. The role of the small GTPase Rab31 in cancer. J Cell Mol Med. 2015;19(1):1-10. doi:10.1111/jcmm.12403

56. Zhang ZY, Lu M, Liu ZK, et al. Rab11a regulates MMP2 expression by activating the PI3K/AKT pathway in human hepatocellular carcinoma cells. Pathol Res Pract. 2020;216(9):153046. doi:10.1016/j. prp.2020.153046

57. Loewith R, Hall MN. Target of rapamycin (TOR) in nutrient signaling and growth control. Genetics. 2011;189(4):1177-1201.

58. Xu BH, Li XX, Yang Y, et al. Aberrant amino acid signaling promotes growth and metastasis of hepatocellular carcinomas through Rab1A-dependent activation of mTORC1 by Rab1A. Oncotarget. 2015;6(25):20813-20828. doi:10.18632/oncotarget.5175

59. Barbieri MA, Roberts RL, Gumusboga A, et al. Epidermal growth factor and membrane trafficking. EGF receptor activation of endocytosis requires Rab5a. J Cell Biol. 2000;151(3):539-550. doi:10.1083/ jcb.151.3.539

60. Geng D, Zhao W, Feng Y, Liu J. Overexpression of Rab25 promotes hepatocellular carcinoma cell proliferation and invasion. Tumour Biol. 2016;37:7713-7718. doi:10.1007/s13277-015-4606-5

61. Shi LW, Zhao ZB, Zhong L, et al. Overexpression of Rab40b promotes hepatocellular carcinoma cell proliferation and metastasis via PI3K/AKT signaling pathway. Cancer Manag Res. 2020;12:10139-10150. doi:10.2147/CMAR.S255870

62. Dong WW, Mou Q, Chen J, Cui JT, Li WM, Xiao WH. Differential expression of Rab27A/B correlates with clinical outcome in hepatocellular carcinoma. World J Gastroenterol. 2012;18(15):1806-1813. doi:10.3748/wjg.v18.i15.1806

63. Wang T, Hong W. Interorganellar regulation of lysosome positioning by the Golgi apparatus through Rab34 interaction with Rab-interacting lysosomal protein. Mol Biol Cell. 2002;13 (12):4317-4332. doi:10.1091/mbc.e02-05-0280

64. Wu J, Lu Y, Qin A, Qiao Z, Jiang X. Overexpression of RAB34 correlates with poor prognosis and tumor progression in hepatocellular carcinoma. Oncol Rep. 2017;38:2967-2974. doi:10.3892/or.2017.5957

65. Su H, Yang JR, Xu T, et al. MicroRNA-101, down-regulated in hepatocellular carcinoma, promotes apoptosis and suppresses tumorigenicity. Cancer Res. 2009;69(3):1135-1142. doi:10.1158/ 0008-5472.CAN-08-2886 
66. Sheng Y, Li J, Zou C, et al. Downregulation of miR-101-3p by hepatitis $\mathrm{B}$ virus promotes proliferation and migration of hepatocellular carcinoma cells by targeting Rab5a. Arch Virol. 2014;159 (9):2397-2410.

67. Dong Z, Qi R, Guo X, et al. MiR-223 modulates hepatocellular carcinoma cell proliferation through promoting apoptosis via the Rab1-mediated mTOR activation. Biochem Biophys Res Commun. 2017;483(1):630-637. doi:10.1016/j.bbrc.2016.12.091

68. Xu WP, Liu JP, Feng JF, et al. miR-541 potentiates the response of human hepatocellular carcinoma to sorafenib treatment by inhibiting autophagy. Gut. 2019;69:1309-1321.

69. Wang B, Dong W, Li X. miR-145-5p acts as a novel tumor suppressor in hepatocellular carcinoma through targeting RAB18. Technol Cancer Res Treat. 2019;18:1533033819850189. doi:10.1177/ 1533033819850189

70. Xue H, Tian GY. MiR-429 regulates the metastasis and EMT of HCC cells through targeting RAB23. Arch Biochem Biophys. 2018;637:48-55. doi:10.1016/j.abb.2017.11.011
71. Chen Z, Wang X, Liu R, et al. KDM4B-mediated epigenetic silencing of miRNA-615-5p augments RAB24 to facilitate malignancy of hepatoma cells. Oncotarget. 2017;8(11):17712-17725. doi:10.18632/oncotarget.10832

72. Kong Q, Fan Q, Ma X, Li J, Ma R. CircRNA circUGGT2 contributes to hepatocellular carcinoma development via regulation of the miR-526b-5p/RAB1A axis. Cancer Manag Res. 2020;12:10229-10241. doi:10.2147/CMAR.S263985

73. Yang L, Peng X, Li Y, et al. Long non-coding RNA HOTAIR promotes exosome secretion by regulating RAB35 and SNAP23 in hepatocellular carcinoma. Mol Cancer. 2019;18(1):78. doi:10.1186/ s12943-019-0990-6

74. Cao SQ, Zheng H, Sun BC, et al. Long non-coding RNA highly up-regulated in liver cancer promotes exosome secretion. World $J$ Gastroenterol. 2019;25(35):5283-5299. doi:10.3748/wjg.v25. i35.5283

\section{Publish your work in this journal}

The Journal of Hepatocellular Carcinoma is an international, peerreviewed, open access journal that offers a platform for the dissemination and study of clinical, translational and basic research findings in this rapidly developing field. Development in areas including, but not limited to, epidemiology, vaccination, hepatitis therapy, pathology and molecular tumor classification and prognostication are all considered for publication. The manuscript management system is completely online and includes a very quick and fair peer-review system, which is all easy to use. Visit http://www.dovepress.com/ testimonials.php to read real quotes from published authors. 